\title{
Retraction note: Assessment of human biomonitoring and DNA microarray analysis in the vicinity population on an industrial complex
}

\author{
Kyoung-Ho Lee, Eun-Kyung Chung, Jeong-Suk Moon, Suk-Woo Nam, \\ Mi-Young Lee \& Bu-Soon Son
}

Retraction Note : Mol Cell Tox (2011) 7:207-219.

DOI: $10.1007 / \mathrm{s} 13273-011-0027-8$

The editors of Molecular \& Cellular Toxicology and the publisher want to notify the readers of a retraction of the article entitled "Assessment of human biomonitoring and DNA microarray analysis in the vicinity population on an industrial complex" by Lee et al. (2011) 7:207-219.

It was brought to our attention that this article was substantially similar to the article "Biomonitoring of urinary metals in a population living in the vicinity of industrial sources: A comparison with the general population of Andalusia, Spain" published in Science of the Total Environment by Aguilera et al. (2008) 407:669-678. After an internal investigation, the Committee of Research and Publication Ethics for the Molecular \& Cellular Toxicology decided to retract the article by Lee et al. from Molecular \& Cellular Toxicology. All the authors agreed to this retraction.

The Editors of Molecular \& Cellular Toxicology (C) The Korean society of Toxicogenomics and Toxicoproteomics and Springer 2013 